ARTICLE

DOI: 10.1038/s41467-018-05262-w

\title{
Introduction of pathogenic mutations into the mouse Psen1 gene by Base Editor and Target-AID
}

Hiroki Sasaguri (1,2, Kenichi Nagata1, Misaki Sekiguchi', Ryo Fujioka', Yukio Matsuba', Shoko Hashimoto', Kaori Sato ${ }^{1}$, Deepika Kurup ${ }^{1,3}$, Takanori Yokota ${ }^{2}$ \& Takaomi C. Saido (iD ${ }^{1}$

Base Editor (BE) and Target-AID (activation-induced cytidine deaminase) are engineered genome-editing proteins composed of Cas9 and cytidine deaminases. These base-editing tools convert $\mathrm{C}: \mathrm{G}$ base pairs to $\mathrm{T}: \mathrm{A}$ at target sites. Here, we inject either BE or Target-AID mRNA together with identical single-guide RNAs (sgRNAs) into mouse zygotes, and compare the base-editing efficiencies of the two distinct tools in vivo. BE consistently show higher base-editing efficiency (10.0-62.8\%) compared to that of Target-AID (3.4-29.8\%). However, unexpected base substitutions and insertion/deletion formations are also more frequently observed in BE-injected mice or zygotes. We are able to generate multiple mouse lines harboring point mutations in the mouse presenilin 1 (Psen1) gene by injection of BE or TargetAID. These results demonstrate that BE and Target-AID are highly useful tools to generate mice harboring pathogenic point mutations and to analyze the functional consequences of the mutations in vivo.

\footnotetext{
${ }^{1}$ Laboratory for Proteolytic Neuroscience, RIKEN Center for Brain Science, Wako-shi, Saitama 351-0198, Japan. ${ }^{2}$ Department of Neurology and Neurological Science, Graduate School of Medicine, Tokyo Medical and Dental University, Bunkyo-ku, Tokyo 113-8510, Japan. ${ }^{3}$ Department of Molecular and Cellular Biology, Harvard University, Cambridge, MA 02138, USA. These authors contributed equally: Hiroki Sasaguri, Kenichi Nagata. Correspondence and requests for materials should be addressed to H.S. (email: hiroki.sasaguri@riken.jp) or to T.C.S. (email: takaomi.saido@riken.jp)
} 
W ith the rapid progress of genetic analyses using human tissues, the number of identified pathogenic mutations has increased drastically. However, significant challenges remain for the functional consequences of these mutations to be explored, not only because the number of patients with specific mutations is often small, but also because the direct evaluation of humans is impractical. In addition, clinically reported mutations are not always related to the disease ${ }^{1}$. Alzheimer's disease (AD), the most common type of dementia, is no exception when it comes to confronting such challenges. Over 200 mutations in the presenilin 1 (PSEN1) gene and more than 50 mutations in the amyloid precursor protein (App) gene have been identified as pathogenic familial $\mathrm{AD}$ (fAD) mutations (Alzforum, http://www.alzforum.org). Most of these are missense mutations with unidentified functional consequences in vivo. Introducing disease-related point mutations into research animals is one of the most reliable ways to elucidate the functional consequences of mutations and the etiology of hereditary diseases. However, generating animal models with such mutations by conventional embryonic stem cell-based gene targeting technology is timeconsuming and requires considerable resources ${ }^{2}$. Novel geneediting technologies such as TALEN and CRISPR/Cas9 overcome these problems, but undesired insertion/deletion (indel) frequently occurs. This is because the non-homologous end-joining pathway, in which the introduced DNA double-strand breaks (DSBs) are repaired by joining the two ends together with random indels, competes with homology-directed repair (HDR), in which the DSBs are rebuilt with template homologous DNA strands carrying the desired mutations. The efficiency of HDR is not always high enough to generate disease models ${ }^{3}$. Furthermore, researchers need to design template DNA for each different mutation.

Recently, novel CRISPR-Cas9-based base-editing technology has been established, in which catalytically deactivated Cas9 (dCas9) or Cas9 nickase (nCas9) is fused with cytidine deaminases to convert C:G base pairs to T:A pairs at target sites with a reduced rate of indel formation in the presence of single-guide RNAs (sgRNAs). Base Editor (BE) is a fusion protein of Streptococcus pyogenes Cas9 (SpCas9) and rat APOBEC1 (apolipoprotein B mRNA-editing enzyme, catalytic polypeptide-like 1$)^{4}$, while Target-AID (activation-induced cytidine deaminase) is composed of SpCas9 and sea lamprey PmCDA1 (Petromyzon marinus cytosine deaminase 1$)^{5}$. In addition to the original $\mathrm{BE}$, mutant versions of BE or BE with Staphylococcus aureus Cas9 (SaCas9) have been generated, in which the proto-spacer adjacent motif (PAM) sequences have been changed from the NGG of the original SpCas $9^{6}$. BE converts Cs at positions 4-8 of the sgRNA target sequences, whereas Target-AID substitutes Cs at positions $2-4$, counting the PAM as positions 21-23. Both these baseediting tools have been reported to convert target bases with high efficiency in vitro ${ }^{4,5}$. More recently, BE has been used to introduce point mutations in mice ${ }^{7,8}$, zebrafish ${ }^{8,9}$, and human zygotes ${ }^{10,11}$. Although Target-AID has been shown to induce mutations in crop plants ${ }^{12}$, it remains unclear whether it works in mammals.

Here we introduced either BE or Target-AID mRNA together with identical sgRNAs that targeted the Psen1 or App gene into mouse zygotes. Multiple animal models were generated with a number of distinct disease-related and disease-unrelated point mutations, thereby enabling the functional consequences of these mutations to be evaluated in vivo.

\section{Results}

Editing efficiency of BE and Target-AID in mouse zygotes. We first took advantage of the overlap of targetable $\mathrm{C}$ in the 4th position of sgRNAs in BE and Target-AID to design an sgRNA that could be used with both base-editing tools. We found that an sgRNA targeting Psen1-P436 had a C in the 4th position (Fig. 1a). We injected sgRNA-Psen1-P436 (60 ng/ $/$ l) (Fig. la, b) together with either BE or Target-AID mRNA $(200 \mathrm{ng} / \mu \mathrm{l})$ into the cytoplasm of C57BL/6 mouse zygotes to generate mutant mice carrying Psen1P436S, which has been identified in fAD patients ${ }^{13}$. We used thirdgeneration $\mathrm{BE}, \mathrm{BE} 3$, that expressed nCas9 fused with rat $\mathrm{APOBEC1}$ and uracil glycosylase inhibitor (UGI) ${ }^{4}$ (hereafter, BE indicates BE3 unless specified otherwise), and Target-AID composed of nCas9 fused with sea lamprey PmCDA1 and $\mathrm{UGI}^{5}$. As expected, BE injection induced efficient conversion of $\mathrm{C}$ to $\mathrm{T}$ at the target sites (Fig. 1c, Table 1, Supplementary Fig. 1a, b). We generated 27 mutant Psen 1 mice by BE out of a total of 43 animals (62.8\%); six mice harbored silent mutations (14.0\%) and 21 mice had missense mutations (48.8\%) (Supplementary Fig. 1e, f). However, because the 5 th $\mathrm{C}$ was more efficiently converted to $\mathrm{T}(39.5 \%)$ than the 4 th $\mathrm{C}(14.0 \%)$, this resulted in the substitution of proline (P) to leucine (L) (CTC or CTT). Thus, mice that harbored Psen1-P436L were generated more frequently (32.6\%) than those carrying Psen1P436S (4.7\%). In addition, unexpected conversion of C to A or G took place not only at the target site but also outside the BE activity window, resulting in the generation of mice with mutations such as Psen1-P436F, Psen1-P436H, Psen1-I437M, or Psen1-S438F (Supplementary Fig. 1a, b, e, f), which have not been reported as fADrelated mutations. Interestingly, some mice harbored two different mutations in different alleles at P436; these included P436S/P436L $(n=1,2.3 \%), \mathrm{P} 436 \mathrm{~L} / \mathrm{P} 436 \mathrm{~F}(n=2,4.7 \%)$, and $\mathrm{P} 436 \mathrm{~F} / \mathrm{P} 436 \mathrm{~V}(n=$ 1, 2.3\%) (Supplementary Fig. 1f). Furthermore, we observed indel formation in $26.9 \%$ of the BE-injected mice (Table 1); these animals were excluded from further analysis because base conversion is sometimes masked by indel signals in the sequencing analysis and because indels in the Psen 1 gene may have affected the function of PS1 in our biochemical analyses.

Following Target-AID injection, 4 out of 34 mice carried mutations in the Psen1 gene (11.8\%), which resulted in Psen1P436S, Psen1-P436L, Psen1-P436I, and Psen1-P436T mutants $(n=$ $1,2.9 \%$ for each mutation) (Fig. 1d, Supplementary Fig. 1g). Consistent with a previous report ${ }^{5}$, the 3 rd $\mathrm{C}$ was most effectively converted to $\mathrm{T}$ in Target-AID-injected mice, resulting in silent mutations in this case (Supplementary Fig. 1c, d). The 4th C was converted to $\mathrm{T}$ with $2.3 \%$ efficiency and to A with $4.7 \%$ efficiency. The 5th and 6th Cs were converted to $\mathrm{T}$ with $2.3 \%$ efficiency, with no other mutations observed for Target-AID. Only 2 out of 36 mice (5.6\%) were found to harbor indels in Target-AID-injected mice (Table 1).

As the target sequence of sgRNA-Psen1-P436 was completely conserved in mouse Psen 2 (chromosome 1), we observed a C to T substitution around Psen2-P428 or indel formation at this position with similar efficiency to Psen1-P436 in BE-injected mice, including the Psen1-P436S founder mouse (\#89) (Supplementary Fig. 2a, b). We also observed substitution of 3rd C to T in the Psen 2 gene in Target-AID-injected mice at low frequency (Supplementary Fig. 2c, d). The mice with Psen 2 mutations were excluded from subsequent biochemical experiments as Psen 2 has also been reported to act as a causative gene for $\mathrm{fAD}^{14}$, and these mutations could have influenced our results.

Next, to assess the off-target effects of BE and Target-AID, we searched for potential off-target sites using Cas-OFFinder ${ }^{15}$ and COSMID ${ }^{16}$. We identified 29 such sites (Supplementary Table 1) and performed targeted deep sequencing in the Psen1P436S founder mice generated by BE or Target-AID. It is worth noting that we did not find any off-target site in the same chromosome as that containing Psen1 (chromosome 12). Our results revealed that the Psen 1-P436S mouse (\#89) which was generated by BE had an off-target mutation at OFF19, which is found in the intron of the fragile histidine triad (Fhit) gene 
(Supplementary Fig. 3a). No apparent increase in mutations at sites other than Fhit and Psen2 was observed, demonstrating the accuracy of the base-editing ability of BE and Target-AID. We confirmed germline transmission of Psen1-P436S in F1 mice in both BE-mediated and Target-AID-mediated lines (Fig. 1c, d). In addition, the undesired mutation in Psen 2 and the off-target mutation in the Fhit gene could be removed in approximately half of the F1 mice that were generated by BE by backcrossing with wild-type mice (Supplementary Fig. 3b).

To further compare the base-editing efficiencies of $\mathrm{BE}$ and Target-AID in vivo, we designed three different sgRNAs that targeted the mouse App gene and injected them with either BE or Target-AID mRNA (Supplementary Fig. 4a-f). The base-editing efficiency of BE was consistently higher (10.0-53.6\%) than that of a

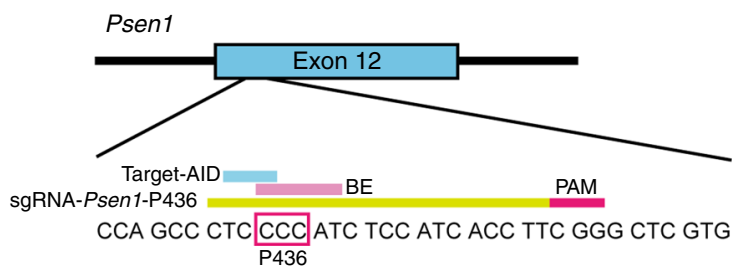

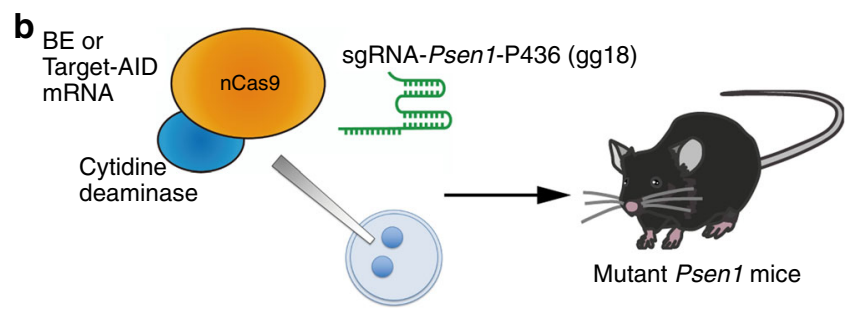

Frequency

C

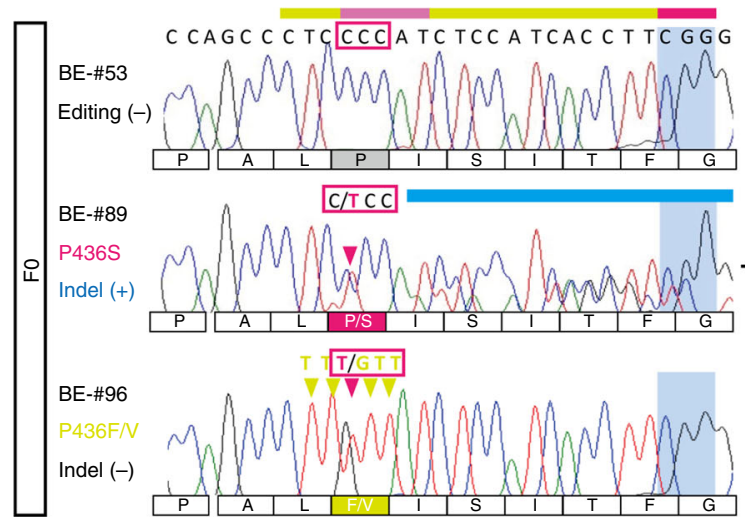

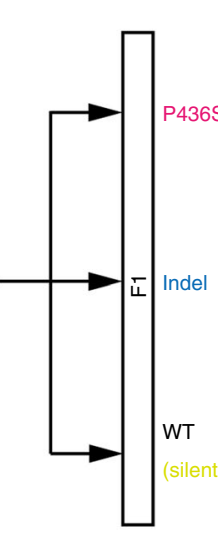
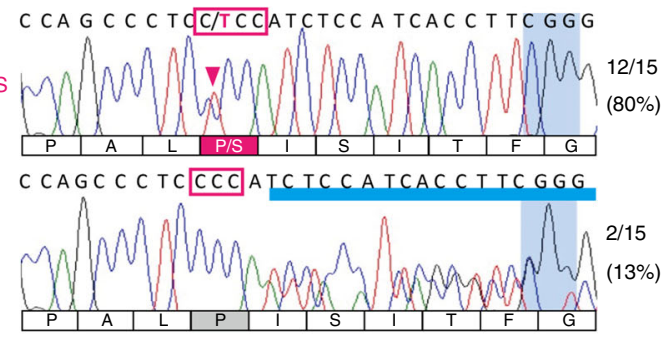

C CA GC CC TC/TCCCATC T CCATCAC C T TCGGG

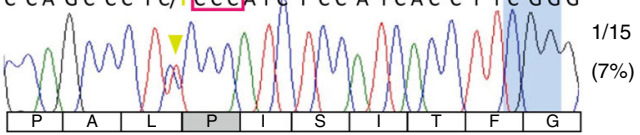

d

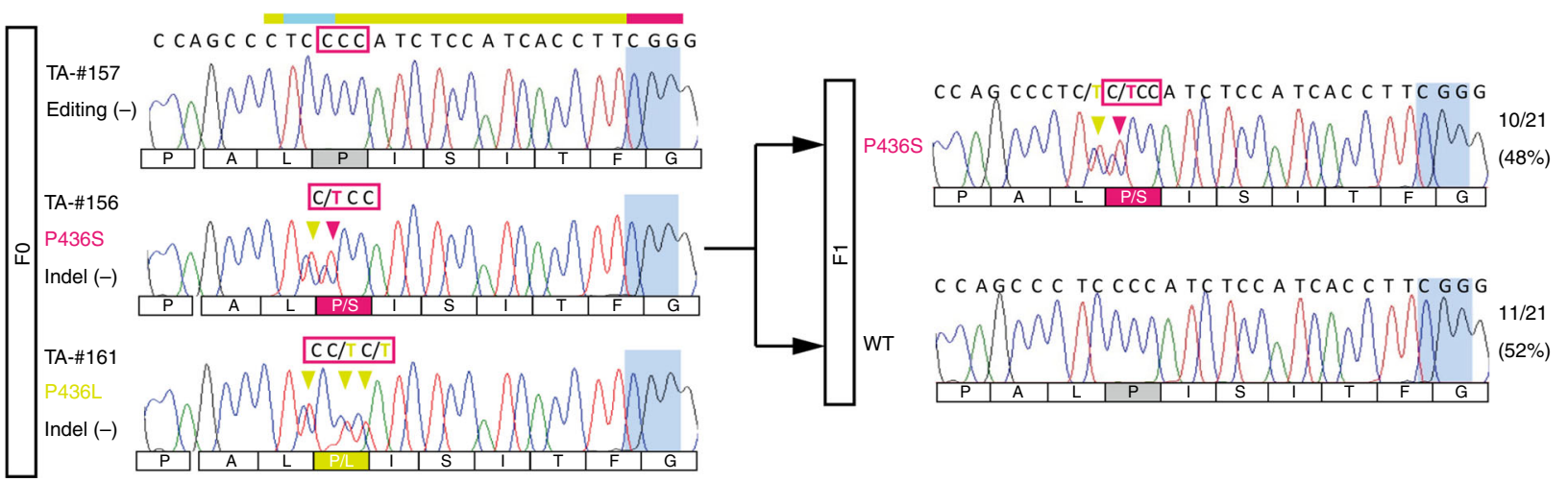

Fig. 1 Generation of Psen1-P436S mice by BE and Target-AID. a Design of sgRNA that targets Psen1-P436. The activity windows of BE (pink) and TargetAID (light blue) are indicated. b Schematic representation of the injection of mouse zygotes for the generation of Psen1-P436S mice. BE or Target-AID mRNA was injected together with sgRNA-Psen1-P436 into the cytoplasm of mouse zygotes to generate mutant Psen1 mice. c, d Sanger sequencing chromatograms in BE-injected (c) and Target-AID-injected (d) mice. Magenta arrowheads indicate expected substitutions, while green arrowheads indicate undesired substitutions, and blue bars indicate indels. The panels on the right represent Sanger sequencing chromatograms of F1 mice generated from the \#89 (BE-injected F0) mouse (c) or the \#156 (Target-AID-injected FO) mouse (d). The column on the right indicates the frequency of each genotype in the F1 generation

Table 1 Summary of the zygotes used and mutant mice obtained after BE or Target-AID injections with sgRNA-Psen1-P436. Nine BE-injected mice and two Target-AID-injected mice were excluded to calculate frequency of substitutions due to existence of indel signals

\begin{tabular}{|lllllll} 
& sgRNA & $\begin{array}{l}\text { No. of injected } \\
\text { zygotes }\end{array}$ & $\begin{array}{l}\text { No. of neonates } \\
\text { (\%) }\end{array}$ & $\begin{array}{l}\text { Frequency of indel } \\
\text { (\%) }\end{array}$ & $\begin{array}{l}\text { Frequency of } \\
\text { substitution (\%) }\end{array}$ & $\begin{array}{l}\text { Frequency of desired } \\
\text { substitution (\%) }\end{array}$ \\
\hline BE & Psen1-P436 & 200 & $52(26.0 \%)$ & $14 / 52(26.9 \%)$ & $27 / 43(62.8 \%)$ & $2 / 43(4.7 \%)$ \\
Target-AID & Psen1-P436 & 150 & $36(24.0 \%)$ & $2 / 36(5.6 \%)$ & $4 / 34(11.8 \%)$ & $1 / 34(2.9 \%)$ \\
\hline
\end{tabular}


Target-AID (3.4-29.8\%) in each of the three targeted regions. However, unexpected substitutions or indel formation were also observed more frequently in BE-injected zygotes (Supplementary Table 2). These results indicate that $\mathrm{BE}$ has a high base-editing efficiency but with less precision, whereas Target-AID edits bases less efficiently but with relatively high accuracy.

Phenotypes of BE-generated and Target-AID-generated Psen1P436S mice. PS1 comprises the catalytic component of the $\gamma$ secretase complex, which cleaves type I transmembrane proteins, including $\mathrm{APP}^{17}$. Several mutant Psen 1 knock-in mouse lines were previously reported ${ }^{18-25}$, and some mice showed aborted maturation of PS1 protein and alteration in processing of its substrates. To analyze the functional consequence of Psen1-P436S mutation in vivo, we first evaluated the viability of homozygous Psen1-P436S neonates because some Psen1 mutations cause embryonic/perinatal lethality or developmental defects when the mutations exist in a homozygous state. The homozygous mutant mice appeared viable and indistinguishable from wild-type littermates (Supplementary Fig. 5a). Western blot analysis indicated that expression levels of $\mathrm{N}$-terminal and C-terminal fragments (NTFs and CTFs) of PS1 protein remained unaltered in heterozygous or homozygous mutant mice (Supplementary Fig. 5b).

CTF- $\beta$, which is generated by proteolytic processing of APP by $\beta$-secretase ( $\beta$-site APP cleaving enzyme 1 or BACE1), is one of the substrates of $\gamma$-secretase. $\gamma$-Secretase first cleaves CTF- $\beta$ by an endopeptidase-like cleavage ( $\varepsilon$-cleavage) to produce long forms of $\beta$-amyloid $(A \beta)$ peptide, $A \beta_{48}$ and $A \beta_{49}$ (Fig. 2a) ${ }^{26}$. Subsequently, $\gamma$-secretase trims these long $A \beta s$ by a carboxypeptidase-like cleavage $(\gamma$-cleavage) to generate smaller $\mathrm{A} \beta$ species of various lengths. The profile of $\mathrm{A} \beta$ peptide production is reported to be altered in fAD patients: $A \beta_{40}$, which is composed of 40 amino acids, is the predominant species in healthy subjects and tends not to aggregate. In contrast, longer $A \beta$ s such as $A \beta_{42}$ and $A \beta_{43}$, which exhibit higher pathogenicity and are prone to aggregation, are increased in the fAD brain ${ }^{27}$. Western blot analysis revealed that full-length APP and CTF- $\beta$ were not altered in the heterozygous Psen1-P436S mice (Fig. 2b, Supplementary Figs. 6, 7). Next, we performed enzyme-linked immunosorbent assay (ELISA) for $A \beta_{40}$ and $A \beta_{42}$ in heterozygous Psen1-P436S mouse brains (Fig. 2c-h). A $\beta_{40}$ was decreased and $A \beta_{42}$ was increased in the guanidine hydrochloride $(\mathrm{GuHCl})$-soluble fractions of Psen1-P436S lines generated by $\mathrm{BE}$ or Target-AID (Fig. $2 \mathrm{f}-\mathrm{h}$ ), resulting in an approximately 1.7 -fold increase in the $A \beta_{42} / A \beta_{40}$ ratio. This increased $A \beta_{42} / A \beta_{40}$ ratio was reproduced in the Tris- $\mathrm{HCl}$ buffered saline-soluble fraction of the mutant lines (Fig. $2 \mathrm{c}-\mathrm{e}$ ). These results suggest that introduction of Psen 1-P436S in mice alters the carboxypeptidase-like cleavage ( $\gamma$-cleavage) activity of $\gamma$-secretase. No age-dependent increase of A $\beta$ (Supplementary Fig. 5c-f) nor amyloid depositions (Supplementary Fig. 5g) in the brain was observed in Psen1-P436S mice. Although these results are consistent with previously reported Psen 1 mutation knock-in mice ${ }^{19,21,24,25}$, further characterization is required to demonstrate the validity of utilizing Psen1-P436S mice for AD research. Interestingly, the biochemical profile of $A \beta$ production in the Psen 1-P436L founder mice that harbored P436L alleles at $35-52 \%$ by mosaicism was similar to that of the Psen1-P436S animals (Supplementary Fig. 8), indicating that the fADunrelated mutation had a similar effect to Psen1-P436S on PS1 functions. These results demonstrate that the BE-mediated and Target-AID-mediated generation of mutant mice is an effective way to analyze the functional consequences of disease-related and disease-unrelated point mutations in vivo.
Efficient generation of multiple mutant mouse lines by BE. Next, we capitalized on the flexible character of BE, which can edit multiple bases with variable patterns, and sought to generate multiple mouse lines that harbor different point mutations. We found that Psen1-P117 is a suitable target for this purpose, because the original CCA (P) can be converted to TCA (S), CTA (L), or TTA (L), and because both Psen1-P117S and Psen1-P117L have been reported to be pathogenic fAD mutations (Fig. 3a). To target Psen1-P117, we used a VQR (D1135V, R1335Q, and T1337R)-BE variant in which the PAM sequence is changed from NGG to $\mathrm{NGA}^{6}$. We injected sgRNA-Psen1-P117 (30 ng/ $\left.\mu \mathrm{l}\right)$ together with VQR-BE mRNA $(200 \mathrm{ng} / \mu \mathrm{l})$ into the cytoplasm of C57BL/6J zygotes (Fig. 3b). In this series, we prepared two sgRNAs of different length, as the length could affect the baseediting efficiency in a manner similar to that of other modified CRISPR/Cas9 tools ${ }^{28}$. SgRNA (gg20) has a 20-base target sequence with two additional mismatched Gs upstream of the target; sgRNA (gg18) has 18 bases of target sequence with two additional mismatched Gs. Sequencing analysis in these mice $(n=77)$ revealed that the targeted Cs were efficiently substituted to T. Consequently, we obtained Psen1-P117S $(n=12,15.6 \%)$ and Psen1-P117L $(n=27,35.1 \%)$ mice at high frequency (Fig. $3 \mathrm{c}$, Table 2). In addition, unexpected substitutions resulted in the generation of Psen1-T116I $(n=1,1.3 \%), \operatorname{Psen} 1-\mathrm{T} 116 \mathrm{~N}(n=2$, 2.6\%), Psen1-P117A $(n=2,3.9 \%)$, and Psen1-P117R $(n=1$, $1.3 \%)$ mutations, which also cause fAD (Supplementary Fig. 9). We also obtained mice harboring fAD-unrelated mutations, such as Psen1-P117Q $(n=8,10.4 \%)$ and Psen1-P117T $(n=3,3.9 \%)$ (Supplementary Fig. 9). The base-editing efficiency was $80.0 \%$ with sgRNA (gg20) and $37.8 \%$ with sgRNA (gg18), while indel formation was $25.9 \%$ with sgRNA (gg20) and $16.3 \%$ with sgRNA (gg18) (Table 2). When we performed ELISA for A $\beta$ using brain samples from the heterozygous Psen 1-P117L mice, we found that they showed increases in $A \beta_{42}$ and in the $A \beta_{42} / A \beta_{40}$ ratio, both in the Tris-HCl-buffered saline-soluble fraction and $\mathrm{GuHCl}$-soluble fraction (Fig. 3d-i). Western blot analysis revealed that fulllength APP and CTF- $\beta$ were not altered in these heterozygous Psen1-P117L mice (Supplementary Fig. 6), suggesting that the $\mathrm{P} 117 \mathrm{~L}$ mutation in the PS1 protein causes alteration of carboxypeptidase-like cleavage $(\gamma$-cleavage) activity of $\gamma$-secretase, similar to that of P436S.

\section{Discussion}

In our experiments, BE and Target-AID were able to introduce point mutations with remarkably high efficiency in vivo compared to conventional gene-targeting or CRISPR/Cas9-based knock-in technologies. Importantly, our mutant Psen 1 mice generated by BE or Target-AID recapitulated the features of previously reported mutant Psen 1 knock-in mice, suggesting that these base-editing tools could be highly useful to analyze the functional consequences of the introduced mutations in vivo. In addition to the high editing efficiencies, these base-editing tools have several advantages over conventional methods; no requirement of donor DNAs and introduction of silent mutations to prevent sequential cleavage by Cas9, and simultaneous generation of multiple mutant lines, especially in the cases where BE is used. Although there are some limitations to using base editing, recent advances in this field are solving some of these issues: evolved versions of the tools dramatically increase the number of targetable regions ${ }^{29,30}$, and recently established adenine $\mathrm{BE}$, which can convert A:T pairs to $\mathrm{G}: \mathrm{C}$ pairs, partially cancels the restrictions for the requirement of the PAM sequence ${ }^{29}$. Base-editing tools should thus facilitate exploration of the unidentified functional consequences of each pathogenic mutation. 
a

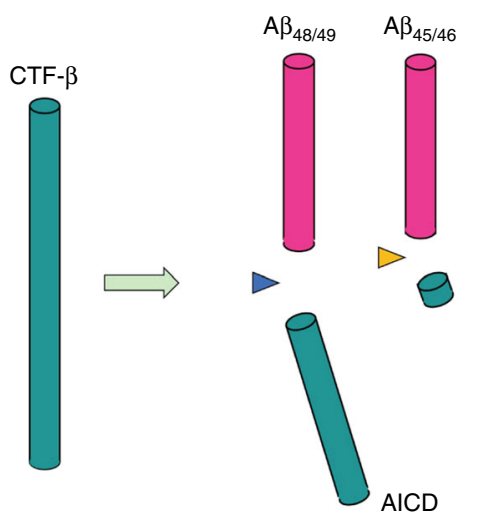

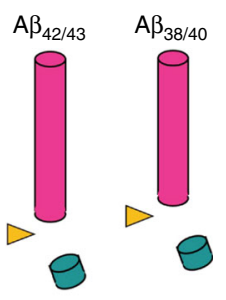

$\triangle \varepsilon$-cleavage

$\triangleleft \gamma$-cleavage b

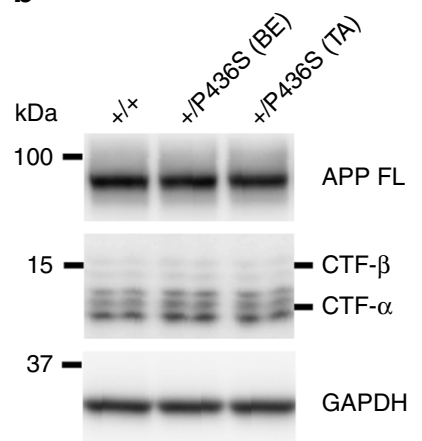

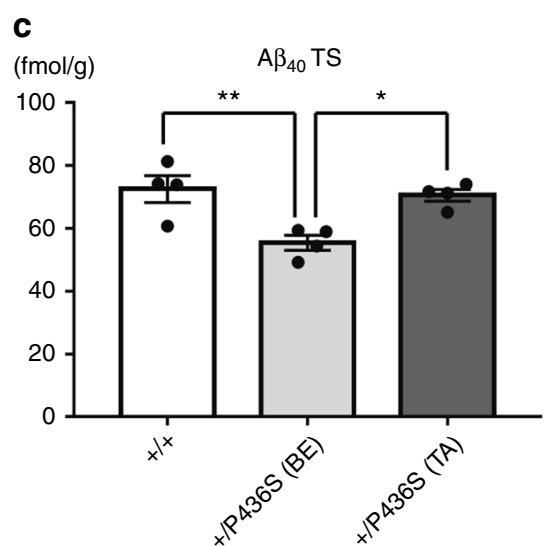

f

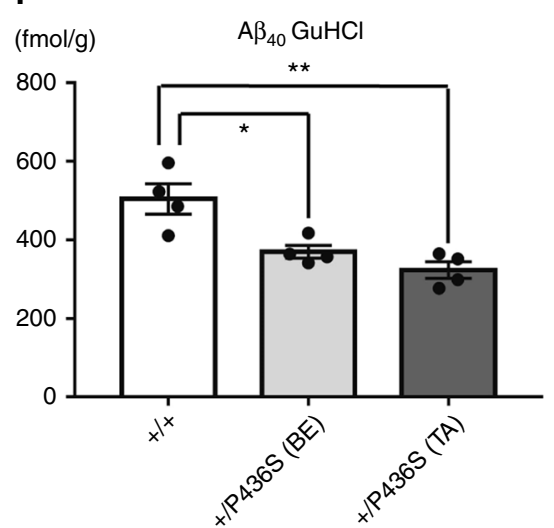

d

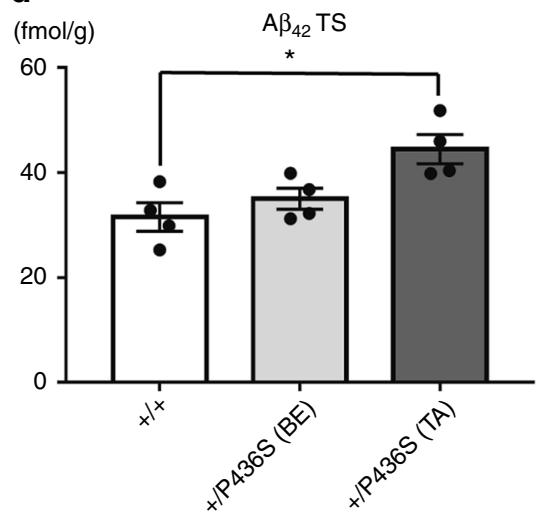

g

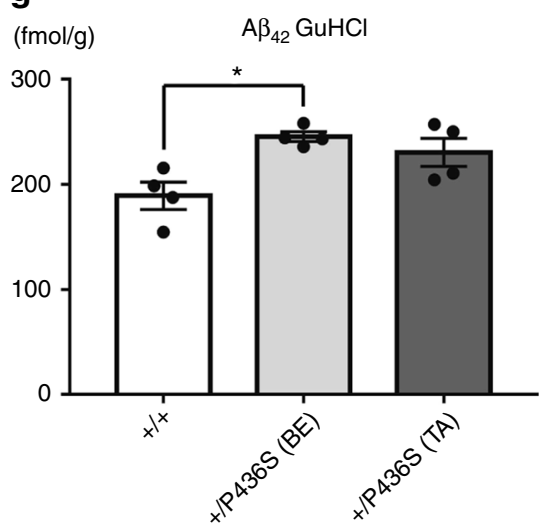

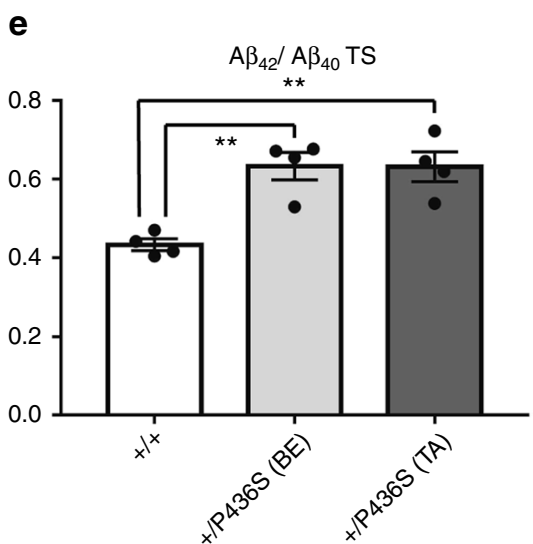

h

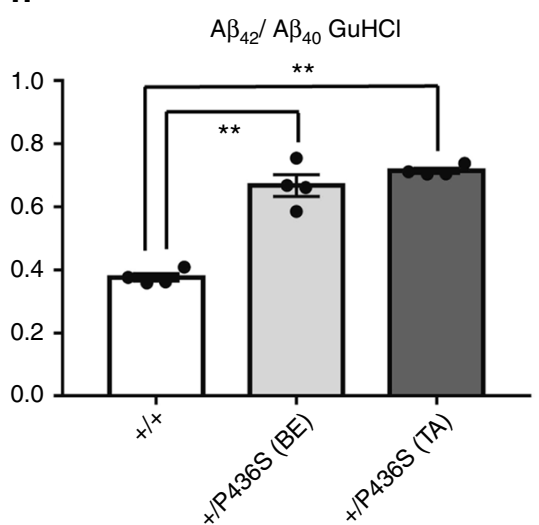

Fig. 2 Phenotypic analysis of Psen1-P436S mice generated by BE and Target-AID. a Sequential processing of APP CTF- $\beta$ by $\gamma$-Secretase. CTF- $\beta$ is first cleaved by an endopeptidase-like cleavage ( $\varepsilon$-cleavage) to produce $A \beta_{48}$ and $A \beta_{49}$, and APP intracellular domain (AICD). Subsequently, $\gamma$-secretase cleaves $A \beta_{48}$ and $A \beta_{49}$ by a carboxypeptidase-like cleavage ( $\gamma$-cleavage) to generate smaller $A \beta$ species. $\mathbf{b}$ Western blot analysis of the full-length (FL) and CTFs of APP in the Psen1-P436S mouse brain. Western blot analysis using anti-APP FL (22C11) and CTFs (APP-CTF) was performed using brain samples of 3-month-old heterozygous Psen1-P436S mice generated by BE or Target-AID. The full-length images are shown in Supplementary Fig. 7. c-h A $\beta$ ELISA of Psen1-P436S mouse brains. Cortices from 3-month-old heterozygous Psen1-P436S mice generated by BE or Target-AID and littermate wild-type mice ( $n=$ 4 for each group) were homogenized and fractionated into Tris- $\mathrm{HCl}$-buffered saline-soluble (TS) (c-e) and guanidine- $\mathrm{HCl}$-soluble $(\mathrm{GuHCl})$ fractions (f-h) and subjected to ELISAs for $A \beta_{40}$ and $A \beta_{42} .{ }^{\star} P \leq 0.05$ and ${ }^{\star \star} P \leq 0.01$ (one-way ANOVA followed by Tukey's post hoc analysis). WT: wild type, BE: Base Editor 3, TA: Target-AID. Data represent mean \pm s.e.m. Genotypes of each mouse are shown in Supplementary Table 3. The information on the sex and age of the mice is provided in Supplementary Table 9

In our study, BE unexpectedly induced indel formation in mouse zygotes at high frequency $(6.7-35.6 \%$, Table 1, 2, Supplementary Table 2) compared to the previous in vitro studies (average $1.1 \%^{4}$ ). The reason for this discrepancy is unclear; however, Kim et al. also reported indel formation in mice generated by $\mathrm{BE}$ at a similar frequency to ours (one out of nine Dmdtargeted mice $(11.1 \%)$ and two out of seven Tyr-targeted mice
$(28.6 \%))^{7}$. The genome-editing or DNA repair mechanism in vivo might be different from those present in cultured cells. BE4, the next generation after BE3 that has an additional UGI in its Cterminus, is reported to induce more precise base conversion compared to $\mathrm{BE}^{31}$. It is therefore possible that base excision repair initiated by uracil DNA glycosirase may be responsible for causing DSB and subsequent indel formation. 
a

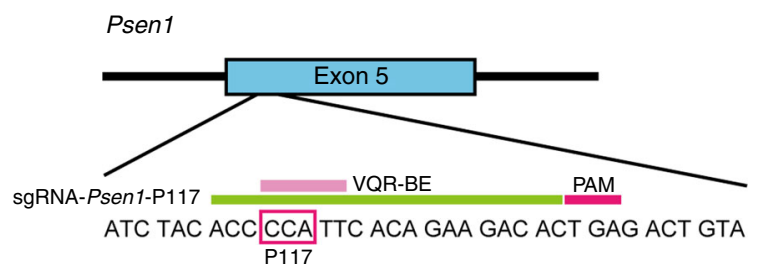

C
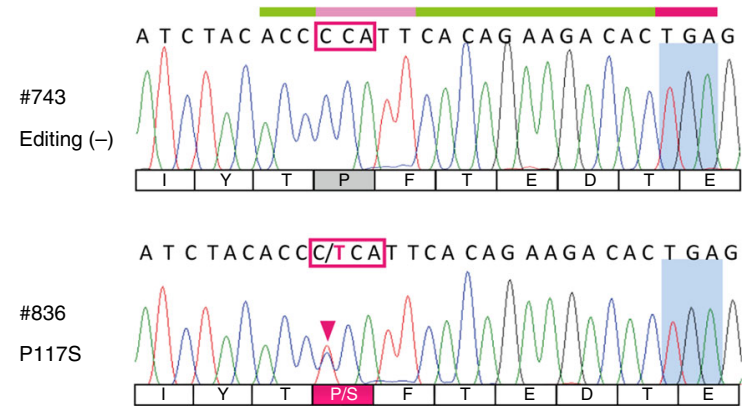

d

$(\mathrm{fmol} / \mathrm{g})$

$\mathrm{A} \beta_{40}$ TS

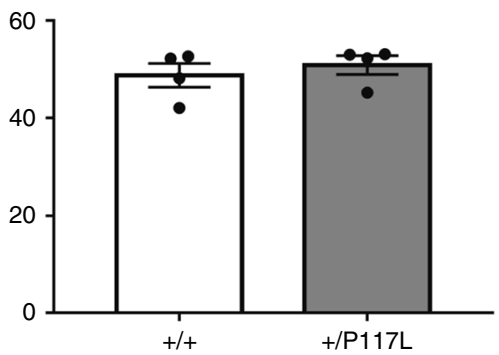

g

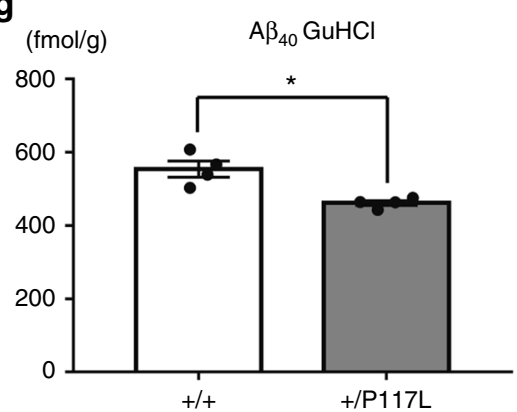

e

(fmol/g)

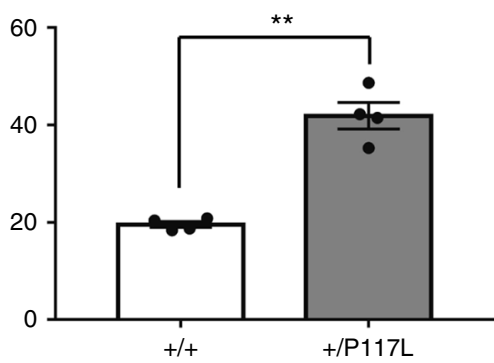

h

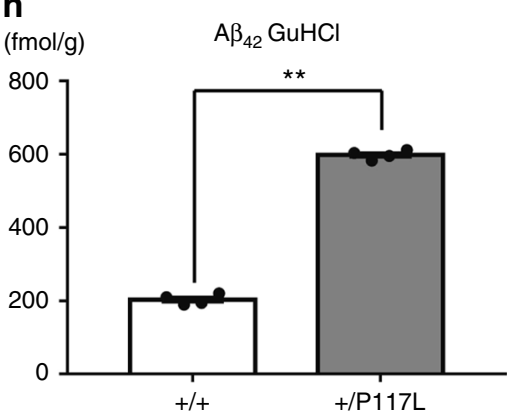

b
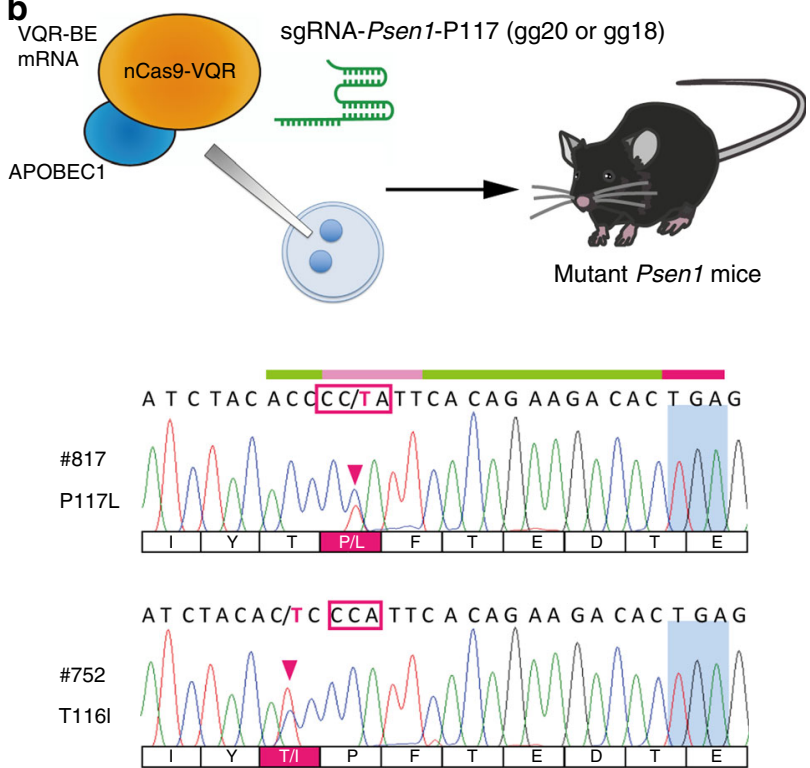

f
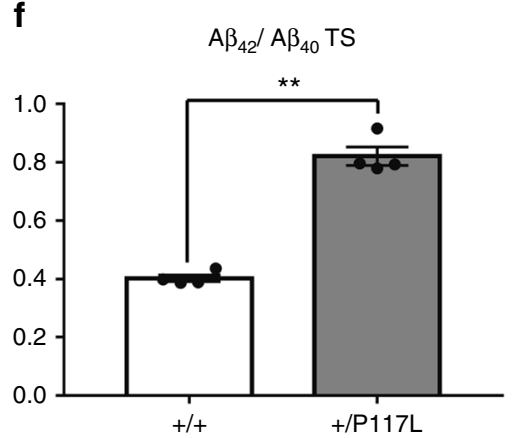

i

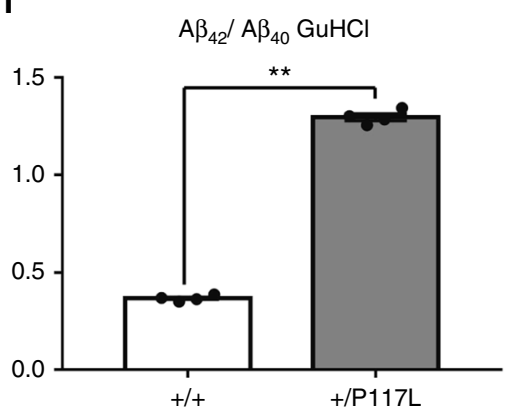

Fig. 3 Generation of mutant Psen1-P117 mice by VQR-BE. a Design of sgRNA that targets Psen1-P117. The target region of VQR-BE (pink) is indicated. b Schematic representation of the injection of mouse zygotes for the generation of mutant Psen1-P117 mice. VQR-BE mRNA was injected together with sgRNA-Psen1-P117 into the cytoplasm of mouse zygotes to generate mutant Psen1 mice. c Sanger sequencing chromatograms of VQR-BE-injected mice. Magenta arrowheads indicate substitutions that result in fAD-related mutations. d-i A $\beta$ ELISA of Psen1-P117L mouse brains. Cortices from 3-month-old heterozygous Psen1-P117L mice generated by VQR-BE and littermate wild-type mice ( $n=4$ for each group) were homogenized and fractionated into Tris$\mathrm{HCl}$-buffered saline-soluble (TS) and guanidine- $\mathrm{HCl}$-soluble $(\mathrm{GuHCl})$ fractions and subjected to ELISAs for $A \beta_{40}$ and $A \beta_{42 .}{ }^{\star} P \leq 0.05$ and ${ }^{\star \star} P \leq 0.01$ (Student's two-tailed $t$-test). Data represent mean \pm s.e.m. Genotypes of each mouse are shown in Supplementary Table 3. The information on the sex and age of the mice is provided in Supplementary Table 9

The base-editing efficiency of Target-AID in mouse zygotes was lower than that of $\mathrm{BE}$, not only at the 4 th $\mathrm{C}$ but also at the 3rd C in some cases, the point at which Target-AID could most effectively edit bases (Supplementary Figs. 1, 4). As Target-AID uses sea lamprey PmCDA1 to convert the target bases, the most appropriate temperature for its application is likely to be around $25^{\circ} \mathrm{C}^{5}$, which might explain the low base-editing efficiency in mouse embryos in the present study, which was conducted at $37^{\circ} \mathrm{C}$.
Although the base-editing efficiency of Target-AID was lower than that of $\mathrm{BE}$ in mice, the frequency of undesired conversion and indel formation was also lower. In addition to targeting the 2nd and 3rd Cs in sgRNA sequences, Target-AID might also be a better option than $\mathrm{BE}$ for introducing precise point mutations targeting the 4 th $\mathrm{C}$.

Because mouse $A \beta$ has low amyloidogenic potential that might be caused by the existence of three different amino acids 
Table 2 Summary of the zygotes used and mutant mice obtained after VQR-BE injections with sgRNA-Psen1-P117. Fourteen sgRNA (gg20)-injected mice and six sgRNA (gg18) injected mice were excluded to calculate frequency of substitutions due to existence of indel signals

\begin{tabular}{|c|c|c|c|c|c|c|}
\hline & sgRNA & $\begin{array}{l}\text { No. of injected } \\
\text { zygotes }\end{array}$ & $\begin{array}{l}\text { No. of } \\
\text { neonates (\%) }\end{array}$ & $\begin{array}{l}\text { Frequency of } \\
\text { indel }(\%)\end{array}$ & $\begin{array}{l}\text { Frequency of } \\
\text { substitution (\%) }\end{array}$ & $\begin{array}{l}\text { Frequency of expected } \\
\text { substitution }(\%)\end{array}$ \\
\hline VQR-BE & $\begin{array}{l}\text { Psen1-P117 (gg20) } \\
\text { Psen1-P117 (gg18) }\end{array}$ & $\begin{array}{l}150 \\
150\end{array}$ & $\begin{array}{l}54(36.0 \%) \\
43(28.7 \%)\end{array}$ & $\begin{array}{l}14 / 54(25.9 \%) \\
7 / 43(16.3 \%)\end{array}$ & $\begin{array}{l}32 / 40(80.0 \%) \\
14 / 37(37.8 \%)\end{array}$ & $\begin{array}{l}25 / 40(62.5 \%) \\
10 / 37(27.0 \%)\end{array}$ \\
\hline
\end{tabular}

compared to human $A \beta^{18,32-34}$, it is difficult to recapitulate full features of $\mathrm{AD}$ such as $\mathrm{A} \beta$ plaque formation or behavioral alterations in mice with a single Psen 1 mutation. However, it is noteworthy that our Psen1-P436S and Psen1-P117L mice showed alteration of their $A \beta$ profiles, which is one of the important pathological features of $\mathrm{AD}$, in a heterozygous state similar to human $\mathrm{fAD}$ patients. To further characterize our mice for $\mathrm{AD}$ research, they need to be crossed with mice harboring humanized sequences of $\mathrm{A} \beta$ peptide, such as our App knock-in mice ${ }^{35}$.

In addition to Psen1-P436S and Psen1-P117L, we identified Psen1-P436L as a potential novel pathogenic mutation that has not been previously reported. Interestingly, the Iberian mutation in $A p p(A p p-\mathrm{I} 716 \mathrm{~F})$ was experimentally predicted by Beyreuther and colleagues to be pathogenic many years before it was actually identified as a clinical mutation ${ }^{36,37}$. Screening using base-editing technology could therefore prove valuable for the identification of such unknown pathogenic mutations or protective variants.

\section{Methods}

Animals. All animal experiments were carried out in accordance with the RIKEN Center for Brain Science guidelines for animal experimentation. C57BL/6J and ICR mouse strains were used as embryo donors and foster mothers, respectively. Wildtype C57BL/6 J mice were used when backcrossing the mutant Psen 1 mice. The information on the sex and age of the mice is provided in Supplementary Table 9.

In vitro transcription. pCMV-BE3 (Addgene plasmid \#73021), pBK-VQR-BE3 (Addgene plasmid \#85171), and pcDNA3.1_pCMV-nCas-PmCDA1-ugi pH1gRNA (Addgene plasmid \#79620) were obtained from Addgene. pCMV-BE3 and pBK-VQR-BE3 were gifts from David Liu. pcDNA3.1_pCMV-nCas-PmCDA1-ugi pH1-gRNA (HPRT) (Addgene plasmid \#79620) was a gift from Akihiko Kondo. The mRNA template for BE, VQR-BE, and Target-AID were prepared by PCR using Herculase II Fusion DNA Polymerase (Agilent Technologies, \#600675) with primers as listed in Supplementary Table 4. BE, VQR-BE, and Target-AID mRNAs were synthesized using the mMESSAGE mMACHINE T7 Ultra Transcription Kit (Thermo Fisher Scientific, AM1345). Templates for in vitro transcription of sgRNAs against each of the Psen 1 mutations and the App gene were prepared by PCR using Herculase II Fusion DNA Polymerase with primers as listed in Supplementary Table 5. The sgRNAs were synthesized using the MEGAshortscript T7 Transcription Kit (Thermo Fisher Scientific, AM1354). Transcribed mRNAs and sgRNAs were purified using the MEGAclear Transcription Clean-Up Kit (Thermo Fisher Scientific, AM1908).

Microinjection into mouse zygotes. RNA solutions containing BE, VQR-BE, or Target-AID mRNA (200 ng/ $\mu \mathrm{l})$, and sgRNAs $(60 \mathrm{ng} / \mu \mathrm{l}$ for Psen1-P436, and $30 \mathrm{ng} / \mu \mathrm{l}$ for Psen 1-P117) were injected into the cytoplasm of C57BL/6J zygotes. After incubation at $37^{\circ} \mathrm{C}$ for 1 day, embryos at the 2-cell-stage were transferred to host ICR female mice.

Genotyping. Genomic DNA was extracted from mouse tails. The tail samples were lysed in lysis buffer (10 mM Tris- $\mathrm{HCl}$ ( $\mathrm{pH} 8.5$ ), $5 \mathrm{mM}$ EDTA ( $\mathrm{pH} 8.0), 0.2 \%$ SDS, $200 \mathrm{mM} \mathrm{NaCl}, 20 \mu \mathrm{g} / \mathrm{ml}$ proteinase $\mathrm{K}$ ), and extracted genomic DNA was directed to PCR and subsequent Sanger sequencing using the primers listed in Supplementary Table 6. For the mice harboring multiple mutations, TA cloning using TA PCR Cloning Kit (BioDynamics Laboratory Inc, pTAC-1), single-colony PCR, and Sanger sequencing using M13-BDFw and M13-BDRev primers (Supplementary Table 6) were performed.

Targeted deep sequencing. Off-target sites that accepted up to three mismatches were determined by Cas-OFFinder (http://www.rgenome.net/cas-offinder/) ${ }^{15}$ and COSMID (https://crispr.bme.gatech.edu//) ${ }^{16}$. Target sites were amplified from tail genomic DNA by PCR using Herculase II Fusion DNA Polymerase with the primers listed in Supplementary Tables 7 and 8. The targeted deep sequencing was performed using an Illumina Miseq system.

Enzyme-linked immunosorbent assay. Mouse cortices were homogenized in 50 $\mathrm{mM}$ Tris- $\mathrm{HCl}$ (pH 7.6) containing $150 \mathrm{mM} \mathrm{NaCl}$ (TBS) and the protease inhibitor cocktail complete mini (Roche Diagnostics) with a Teflon-glass homogenizer. After centrifugation at $200,000 \times g$ for $20 \mathrm{~min}$ at $4{ }^{\circ} \mathrm{C}$, the supernatant was collected (TS fraction). The pellet was dissolved in $6 \mathrm{M}$ guanidine- $\mathrm{HCl}$ buffer. The solubilized pellet was centrifuged at $200,000 \times g$ for $20 \mathrm{~min}$ at room temperature and then used as the insoluble fraction ( $\mathrm{GuHCl}$ fraction). The amounts of $\mathrm{A} \beta_{40}$ and $\mathrm{A} \beta_{42}$ in each fraction were determined by A $\beta$ ELISA kit (Wako, 294-62501 and 294-62601), according to the manufacturer's instructions ${ }^{38}$.

Western blot analysis. Homogenates from brain cortices at postnatal day 5 and 3 month-old mice were subjected to sodium dodecyl sulfate-polyacrylamide gel electrophoresis (SDS-PAGE), and transferred electrophoretically to a $0.22-\mu \mathrm{m}$ PVDF membrane. Antibodies against APP (22C11, Millipore, \#MAB348, 1:2000), APP-CTF (Sigma, \#A8717, 1:10,000), GAPDH (TREVIGEN, \#2275-PC-100, 1:5000), PS1-NTF and PS1-CTF (1:2000, kind gifts from Dr. Tomita of Tokyo University) were used ${ }^{39,40}$. Immunoreactive bands on the membrane were visualized with ECL Select (GE Healthcare) and scanned with a LAS-3000mini Lumino Image analyzer (Fujifilm).

Immunohistochemical analysis. Immunohistochemical staining against $\mathrm{A} \beta$ (N1D 1:200) was performed in 9-month-old Psen 1-P436S mice and 24-month-old NL-F mice. After deparaffinization of paraffin-embedded mouse brain sections, antigen retrieval was performed by autoclave treatment $\left(121^{\circ} \mathrm{C}\right.$ for $\left.5 \mathrm{~min}\right)$. The sections were rinsed several times, blocked for $30 \mathrm{~min}$, and then incubated with primary antibody in blocking solution overnight. A biotinylated secondary antibody and tyramide signal amplification (PerkinElmer) were used for detection of amyloid pathology. Photographic data were captured using a NanoZoomer Digital Pathology C9600 (Hamamatsu Photonics)

Statistical analyses. All data are shown as the mean \pm s.e.m. For comparison between two groups, data were analyzed by Student's $t$-test. For comparisons among three groups, we used one-way analysis of variance followed by Tukey's post hoc analysis. The data were collected and processed in a randomized and blinded manner.

Data availability. Sequencing information has been uploaded to DNA Data Bank of Japan (DDBJ) Sequencing Read Archive (DRA) with the accession code DRA007028. The data related to the findings of this study are available from the corresponding authors on request.

Received: 27 January 2018 Accepted: 22 June 2018

Published online: 24 July 2018

\section{References}

1. MacArthur, D. G. et al. Guidelines for investigating causality of sequence variants in human disease. Nature 508, 469-476 (2014).

2. Liu, E. T. et al. Of mice and CRISPR: the post-CRISPR future of the mouse as a model system for the human condition. EMBO Rep. 18, 187-193 (2017).

3. Komor, A. C., Badran, A. H. \& Liu, D. R. CRISPR-based technologies for the manipulation of eukaryotic genomes. Cell 169, 559 (2017).

4. Komor, A. C., Kim, Y. B., Packer, M. S., Zuris, J. A. \& Liu, D. R. Programmable editing of a target base in genomic DNA without double-stranded DNA cleavage. Nature 533, 420-424 (2016).

5. Nishida, K. et al. Targeted nucleotide editing using hybrid prokaryotic and vertebrate adaptive immune systems. Science 353, 6305 (2016).

6. Kim, Y. B. et al. Increasing the genome-targeting scope and precision of base editing with engineered Cas9-cytidine deaminase fusions. Nat. Biotechnol. 35, 371-376 (2017) 
7. Kim, K. et al. Highly efficient RNA-guided base editing in mouse embryos. Nat. Biotechnol. 35, 435-437 (2017).

8. Rees, H. A. et al. Improving the DNA specificity and applicability of base editing through protein engineering and protein delivery. Nat. Commun. 8 , 15790 (2017)

9. Zhang, Y. et al. Programmable base editing of zebrafish genome using a modified CRISPR-Cas9 system. Nat. Commun. 8, 118 (2017)

10. Li, G. et al. Highly efficient and precise base editing in discarded human tripronuclear embryos. Protein Cell 8, 776-779 (2017).

11. Zhou, C. et al. Highly efficient base editing in human tripronuclear zygotes. Protein Cell 8, 772-775 (2017).

12. Shimatani, Z. et al. Targeted base editing in rice and tomato using a CRISPRCas9 cytidine deaminase fusion. Nat. Biotechnol. 35, 441-443 (2017).

13. Palmer, M. S. et al. Pathogenic presenilin 1 mutations (P436S \& I143F) in early-onset Alzheimer's disease in the UK. Hum. Mutat. 13, 256 (1999).

14. Karch, C. M., Cruchaga, C. \& Goate, A. M. Alzheimer's disease genetics: from the bench to the clinic. Neuron 83, 11-26 (2014).

15. Bae, S., Park, J. \& Kim, J. S. Cas-OFFinder: a fast and versatile algorithm that searches for potential off-target sites of Cas9 RNA-guided endonucleases. Bioinformatics 30, 1473-1475 (2014).

16. Cradick, T. J., Qiu, P., Lee, C. M., Fine, E. J. \& Bao, G. COSMID: a web-based tool for identifying and validating CRISPR/Cas off-target sites. Mol. Ther. Nucleic Acids 3, e214 (2014).

17. Li, Y. M. et al. Photoactivated gamma-secretase inhibitors directed to the active site covalently label presenilin 1 . Nature 405, 689-694 (2000).

18. Guo, Q. et al. Increased vulnerability of hippocampal neurons to excitotoxic necrosis in presenilin-1 mutant knock-in mice. Nat. Med. 5, 101-106 (1999).

19. Nakano, Y. et al. Accumulation of murine amyloidbeta 42 in a gene-dosagedependent manner in PS1 'knock-in' mice. Eur. J. Neurosci. 11, 2577-2581 (1999).

20. Siman, R. et al. Presenilin-1 P264L knock-in mutation: differential effects on abeta production, amyloid deposition, and neuronal vulnerability. J. Neurosci. 20, 8717-8726 (2000).

21. Casas, C. et al. Massive CA1/2 neuronal loss with intraneuronal and Nterminal truncated Abeta42 accumulation in a novel Alzheimer transgenic model. Am. J. Pathol. 165, 1289-1300 (2004).

22. Shimojo, M. et al. Enzymatic characteristics of I213T mutant presenilin-1/ gamma-secretase in cell models and knock-in mouse brains: familial Alzheimer disease-linked mutation impairs gamma-site cleavage of amyloid precursor protein C-terminal fragment beta. J. Biol. Chem. 283, 16488-16496 (2008).

23. Saito, T. et al. Potent amyloidogenicity and pathogenicity of Abeta43. Nat. Neurosci. 14, 1023-1032 (2011).

24. Xia, D. et al. Presenilin-1 knockin mice reveal loss-of-function mechanism for familial Alzheimer's disease. Neuron 85, 967-981 (2015).

25. Vidal, R. et al. The Psen1-L166P-knock-in mutation leads to amyloid deposition in human wild-type amyloid precursor protein YAC transgenic mice. FASEB J. 26, 2899-2910 (2012).

26. Tomita, T. \& Iwatsubo, T. Structural biology of presenilins and signal peptide peptidases. J. Biol. Chem. 288, 14673-14680 (2013).

27. Selkoe, D. J. \& Hardy, J. The amyloid hypothesis of Alzheimer's disease at 25 years. EMBO Mol. Med. 8, 595-608 (2016)

28. Dahlman, J. E. et al. Orthogonal gene knockout and activation with a catalytically active Cas9 nuclease. Nat. Biotechnol. 33, 1159-1161 (2015).

29. Gaudelli, N. M. et al. Programmable base editing of $A^{\star} T$ to $G^{*} C$ in genomic DNA without DNA cleavage. Nature 551, 464-471 (2017).

30. Hu, J. H. et al. Evolved Cas9 variants with broad PAM compatibility and high DNA specificity. Nature 556, 57-63 (2018).

31. Komor, A. C. et al. Improved base excision repair inhibition and bacteriophage $\mathrm{Mu}$ Gam protein yields C:G-to-T:A base editors with higher efficiency and product purity. Sci. Adv. 3, eaao4774 (2017).

32. Chui, D. H. et al. Transgenic mice with Alzheimer presenilin 1 mutations show accelerated neurodegeneration without amyloid plaque formation. Nat. Med. 5, 560-564 (1999).

33. Schmitz, C. et al. Hippocampal neuron loss exceeds amyloid plaque load in a transgenic mouse model of Alzheimer's disease. Am. J. Pathol. 164, 1495-1502 (2004).

34. Xu, G. et al. Murine Abeta over-production produces diffuse and compact Alzheimer-type amyloid deposits. Acta Neuropathol. Commun. 3, 72 (2015).

35. Saito, T. et al. Single App knock-in mouse models of Alzheimer's disease. Nat. Neurosci. 17, 661-663 (2014).
36. Guerreiro, R. J. et al. Genetic screening of Alzheimer's disease genes in Iberian and African samples yields novel mutations in presenilins and APP. Neurobiol. Aging 31, 725-731 (2010).

37. Lichtenthaler, S. F. et al. Mechanism of the cleavage specificity of Alzheimer's disease gamma-secretase identified by phenylalanine-scanning mutagenesis of the transmembrane domain of the amyloid precursor protein. Proc. Natl Acad. Sci. USA 96, 3053-3058 (1999).

38. Iwata, N. et al. Presynaptic localization of neprilysin contributes to efficient clearance of amyloid-beta peptide in mouse brain. J. Neurosci. 24, 991-998 (2004).

39. Sato, C., Takagi, S., Tomita, T. \& Iwatsubo, T. The C-terminal P. A. L. motif and transmembrane domain 9 of presenilin 1 are involved in the formation of the catalytic pore of the gamma-secretase. J. Neurosci. 28, 6264-6271 (2008).

40. Tomita, T. et al. C terminus of presenilin is required for overproduction of amyloidogenic Abeta42 through stabilization and endoproteolysis of presenilin. J. Neurosci. 19, 10627-10634 (1999).

\section{Acknowledgements}

We thank Yukiko Nagai for secretarial assistance, and Erika Sasaki and Wakako Kumita (Central Institute for Experimental Animals) for valuable discussions and advice. This work was supported by AMED under Grant Number JP18dm0207001 (Brain Mapping by Integrated Neurotechnologies for Disease Studies (Brain/MINDS)) (T.C.S.), JSPS Grant-in-Aid for Scientific Research (C) Grant Number 18K07402 (H.S.), and the Special Postdoctoral ResearchersProgram from RIKEN (S.H.). Manipulations to embryos, including injections of $\mathrm{BE}$ and Target-AID into zygotes and embryo transfer were performed at the RIKEN CBS-Research Resource Division (RRD). We are grateful to the RIKEN Center for Brain Science RRD for technical help with DNA sequencing analyses. We thank Michael Patterson, Sciencedit, for editing the English in our manuscript.

\section{Author contributions}

H.S., K.N., and T.C.S. conceived the study. H.S. and K.N. designed the experiments. H.S., M.S., R.F., D.K., Y.M., S.H., and K.S. performed the experiments. H.S. and K.N. analyzed the data and prepared the figures. H.S., K.N., S.H., T.Y., and T.C.S. wrote and edited the manuscript. All authors provided feedback and agreed on the final manuscript.

\section{Additional information}

Supplementary Information accompanies this paper at https://doi.org/10.1038/s41467 018-05262-w.

Competing interests: T.C.S. serves as the CEO for RIKEN BIO Co. Ltd., a RIKEN venture based at the Center for Brain Science, Central Building, Wako, Japan. S.H., Y.M., and T.C.S. serve as research scientist, director, and CEO, respectively, for RIKEN BIO Co. Ltd., a RIKEN venture based at the Brain Science Institute, Central Building, Wako, Japan. The remaining authors declare no competing interests.

Reprints and permission information is available online at http://npg.nature.com/ reprintsandpermissions/

Publisher's note: Springer Nature remains neutral with regard to jurisdictional claims in published maps and institutional affiliations.

Open Access This article is licensed under a Creative Commons Attribution 4.0 International License, which permits use, sharing, adaptation, distribution and reproduction in any medium or format, as long as you give appropriate credit to the original author(s) and the source, provide a link to the Creative Commons license, and indicate if changes were made. The images or other third party material in this article are included in the article's Creative Commons license, unless indicated otherwise in a credit line to the material. If material is not included in the article's Creative Commons license and your intended use is not permitted by statutory regulation or exceeds the permitted use, you will need to obtain permission directly from the copyright holder. To view a copy of this license, visit http://creativecommons.org/ licenses/by/4.0/.

(C) The Author(s) 2018 